\title{
On Special Pieces in the Unipotent Variety
}

\author{
Meinolf Geck and Gunter Malle
}

\section{CONTENTS}

1. Introduction

2. The Algorithm

3. An Extension

4. Complex Reflection Groups

Acknowledgements

Electronic Availability

References
This article is the result of experiments performed using computer programs written in the GAP language. We describe an algorithm which computes a set of rational functions attached to a finite Coxeter group W. Conjecturally, these rational functions should be polynomials, and in the case where $W$ is the Weyl group of a Chevalley group $G$ defined over $\mathbb{F}_{q}$, the values of our polynomials at $q$ should give the number of $\mathbb{F}_{q}$-rational points of Lusztig's special pieces in the unipotent variety of $\mathrm{G}$. The algorithm even works for complex reflection groups. We give a number of examples which show, in particular, that our conjecture is true for all types except possibly $B_{n}$ and $D_{n}$.

\section{INTRODUCTION}

Let $G$ be a connected reductive algebraic group defined over some algebraically closed field $k$. Let $X_{G}$ be the partially ordered set of unipotent classes of $G$, where we write $C \leq C^{\prime}$ if and only if $C$ lies in the Zariski closure of $C^{\prime}$. Following Spaltenstein [1982] and Lusztig [1997], we can define a partition of $X_{G}$ into so-called special pieces. To do this, we first have to recall some facts about the Springer correspondence and special characters of Weyl groups. (These facts can be found in [Lusztig 1984, (13.1)] for the case where the characteristic of $k$ is "good" for $G$, and in [Geck and Malle 1999, Theorem 2.1] for the case of "bad" characteristic.)

Let $W$ be the Weyl group of $G$ (with respect to some maximal torus). The Springer correspondence associates with each irreducible character of $W$ a pair $(C, \psi)$, where $C \in X_{G}$ and $\psi$ is an irreducible character of the group of components of the centralizer of an element in $C$. This correspondence is injective but, in general, not surjective. However, it is a fact that all pairs $(C, 1)$ arise in this way. Given $C \in X_{G}$ we denote by $\varphi_{C}$ the irreducible character of $W$ such that $\varphi_{C}$ corresponds to $(C, 1)$.

Recall from [Lusztig 1984, Chap. 4] that the irreducible characters of $W$ are partitioned into families 
and that each family contains a unique special character. Another known fact is that all special characters are of the form $\varphi_{C}$ for some $C \in X_{G}$. A unipotent class $C \in X_{G}$ is called special if the character $\varphi_{C}$ is special.

The required partition of $X_{G}$ is now defined as follows. Each piece of this partition is a union of some unipotent classes of $G$. Two unipotent classes $C, C^{\prime} \in X_{G}$ belong to the same piece if and only if $\varphi_{C}, \varphi_{C^{\prime}}$ belong to the same family of characters of $W$. Since each family contains a unique special character and each special character is of the form $\varphi_{C}$ for some class $C$, we see that each piece of $X_{G}$ contains a unique special unipotent class. These pieces are called the special pieces of $X_{G}$. One of the main results of [Lusztig 1997] asserts that a special piece consists precisely of the unique special unipotent class $C$ in it and all unipotent classes in the closure of $C$ which are not contained in the closure of any strictly smaller special unipotent class.

Now assume that $k$ is an algebraic closure of the finite field $\mathbb{F}_{q}$ (where $q$ is a power of some prime $p$ ) and that $G$ has a split $\mathbb{F}_{q}$-rational structure, with corresponding Frobenius map $F$. Then each unipotent class is $F$-stable and, if $C$ is such a class, there exists a polynomial $f_{C} \in \mathbb{Q}[u]$ (where $u$ is an indeterminate) such that $\left|C^{F^{s}}\right|=f_{C}\left(q^{s}\right)$ for all $s \geq 1$. Note, however, that the classification of unipotent classes is different for different primes $p$. Nevertheless, Lusztig has shown the following surprising result in [Lusztig 1997] (which appeared as a conjecture in [Lusztig 1981a]):

Theorem 1.1 [Lusztig 1997]. Let $W$ be a finite Weyl group. Then there exists a collection of polynomials $\left\{f_{\varphi}\right\} \subseteq \mathbb{Z}[u]$, one for each special character $\varphi$ of $W$, such that the following hold: whenever $G$ is a connected reductive algebraic group with Weyl group $W$ and $F: G \rightarrow G$ is a Frobenius map corresponding to some split $\mathbb{F}_{q}$-rational structure on $G$ (for some prime power $q)$, then $\left|\mathfrak{C}^{F}\right|=f_{\varphi}(q)$ where $\mathcal{C}$ is the special piece corresponding to $\varphi$.

Lusztig's proof is case by case, using some very elaborate counting arguments. This paper arose from an attempt to find a more conceptual proof. We propose a general algorithm for computing the polynomials $f_{\varphi}$. This algorithm even works for complex reflection groups. Several examples of computations will be given. The algorithm was found by experimentation, with the help of programs written in CHEVIE [Geck et al. 1996] or GAP [Schönert et al. 1994]. See also the section on Electronic Availability at the end of this paper.

\section{THE ALGORITHM}

We will describe an algorithm, which takes as input a finite Coxeter system $(W, S)$ and returns a list of polynomials, one for each special character of $W$. This algorithm is a variant of that for computing Green functions, as explained in Shoji [1987].

First, we need to recall the basic definitions of the $a$-invariants and the $b$-invariants of the irreducible characters of $W$ (see [Lusztig 1979] for more background).

Let $V$ be a real vector space and $W \subset \mathrm{GL}(V)$ the standard geometric realization of $W$, where the elements in $S$ are reflections (see [Bourbaki 1968, Chap. V, $\S 4]$ ). Let $u$ be an indeterminate; we define

$$
P_{W}:=\prod_{i=1}^{|S|} \frac{u^{d_{i}}-1}{u-1}
$$

where $d_{1}, d_{2}, \ldots$ are the degrees of $W$. Let $\mathrm{CF}(W)$ be the space of $\mathbb{R}$-valued class functions on $W$, and let $R: \mathrm{CF}(W) \rightarrow \mathbb{R}(u)$ be the map defined by

$$
R(f):=P_{W}(u-1)^{|S|} \frac{1}{|W|} \sum_{w \in W} \frac{\varepsilon(w) f(w)}{\operatorname{det}\left(u \cdot \operatorname{id}_{V}-w\right)}
$$

for $f \in \mathrm{CF}(W)$, where $\varepsilon$ denotes the sign character. Then we have in fact $R(f) \in \mathbb{R}[u]$ and even $R(f) \in \mathbb{Z}[u]$ if $f$ is a character (see [Carter 1985, Proposition 11.1.1]). If $\varphi \in \operatorname{Irr}(W)$, then $R(\varphi)$ is called the fake degree of $\varphi$. The b-invariant of $\varphi$ is defined as the largest $r \geq 0$ such that $u^{r}$ divides $R(\varphi)$ or, equivalently, as the smallest $r \geq 0$ such that $\varphi$ occurs with nonzero multiplicity in the character of the $r$-th symmetric power of the $W$ module $V$.

We define a matrix $\Omega=\left(\omega_{\varphi, \varphi^{\prime}}\right)_{\varphi, \varphi^{\prime} \in \operatorname{Irr}(W)}$ (with entries in $\mathbb{Z}[u]$ ) by

$$
\omega_{\varphi, \varphi^{\prime}}=u^{N} R\left(\varphi \otimes \varphi^{\prime} \otimes \varepsilon\right)
$$

where $N$ is the number of reflections in $W$. We shall need the following result: 
Lemma 2.1 (Lusztig). For any $\varphi, \varphi^{\prime} \in \operatorname{Irr}(W)$, we have

$$
\omega_{\varphi, \varphi^{\prime}}=\delta_{\varphi, \varphi^{\prime}} u^{2 N}+\text { remainder }
$$

where remainder is a linear combination of strictly smaller powers of $u$ and $\delta_{\varphi, \varphi^{\prime}}$ is the Kronecker symbol. Consequently, the determinant of any principal minor of $\Omega$ is nonzero.

Proof. Write $\varphi \otimes \varphi^{\prime} \otimes \varepsilon=\sum_{\varphi^{\prime \prime}} c_{\varphi^{\prime \prime}} \varphi^{\prime \prime}$ where the sum is over all $\varphi^{\prime \prime} \in \operatorname{Irr}(W)$ and $c_{\varphi^{\prime \prime}}$ are nonnegative integers. It is clear that $c_{\varepsilon}=\delta_{\varphi, \varphi^{\prime}}$. Hence

$$
\omega_{\varphi, \varphi^{\prime}}=u^{N} \delta_{\varphi, \varphi^{\prime}} R(\varepsilon)+\sum_{\varphi^{\prime \prime} \neq \varepsilon} c_{\varphi^{\prime \prime}} u^{N} R\left(\varphi^{\prime \prime}\right) .
$$

Now the definition of $R$ shows that $R\left(1_{W}\right)=1$ (where $1_{W}$ denotes the trivial character) and that $R\left(\varphi^{\prime \prime}\right)$ has constant term 0 if $\varphi^{\prime \prime} \neq 1_{W}$. Combining this with the formula in [Carter 1985, Proposition 11.1.2], we conclude that $R(\varepsilon)=u^{N}$ and that $R\left(\varphi^{\prime \prime}\right)$ is a polynomial in $u$ of degree $<N$ if $\varphi^{\prime \prime} \neq \varepsilon$. Thus, the first statement is proved.

Now consider a principal minor of $\Omega$ of size $k$. The diagonal entries of that minor are all monic polynomials of degree $2 N$, and the off-diagonal entries are polynomials in $u$ of degree strictly smaller than $2 N$. This implies that the determinant of that minor is a monic polynomial in $u$ of degree $2 N k$; in particular, it is nonzero.

To define the $a$-invariants, we need the notion of the generic degree of an irreducible character of $W$ (see [Benson and Curtis 1972]). They are defined in terms of the 1-parameter generic Iwahori-Hecke algebra $H$ associated with $(W, S)$. This is an associative algebra over the field $\mathbb{R}\left(u^{1 / 2}\right)$ (where $u^{1 / 2}$ is an indeterminate), with a basis $\left\{T_{w} \mid w \in W\right\}$ such that the following relations hold:

1. $T_{w} T_{w^{\prime}}=T_{w w^{\prime}}$ if $l\left(w w^{\prime}\right)=l(w)+l\left(w^{\prime}\right)$;

2. $T_{s}^{2}=u T_{1}+(u-1) T_{s}$ for $s \in S$.

It is known that the algebra $H$ is split semisimple (see [Lusztig 1981b; Kilmoyer and Solomon 1973; Alvis and Lusztig 1982]) and that the values of the irreducible characters of $H$ at basis elements $T_{w}$ lie in $\mathbb{R}\left[u^{1 / 2}\right]$. By Tits' Deformation Theorem (see [Curtis and Reiner 1987, $\S 68 \mathrm{~A}$ ]), we have in fact a bijection between the irreducible characters of $H$ and those of $W$. If $\varphi$ is an irreducible character of $W$, we denote by $\varphi_{u}$ the corresponding character of
$H$; this correspondence is uniquely determined by the condition that $\theta\left(\varphi_{u}\left(T_{w}\right)\right)=\varphi(w)$ for all $w \in W$, where $\theta: \mathbb{R}\left[u^{1 / 2}\right] \rightarrow \mathbb{R}, u^{1 / 2} \mapsto 1$. The algebra $H$ carries a symmetrizing trace $\tau: H \rightarrow \mathbb{R}(u)$ given by $\tau\left(T_{1}\right)=1$ and $\tau\left(T_{w}\right)=0$ for $1 \neq w \in W$. Using a specialization argument as above, we see that every irreducible character of $H$ appears in $\tau$ with nonzero multiplicity. The generic degrees $D_{\varphi}$ associated with the irreducible characters $\varphi$ of $W$ can now be defined by the equation:

$$
\tau=\sum_{\varphi \in \operatorname{Irr}(W)} \frac{P_{W}}{D_{\varphi}} \varphi_{u} .
$$

By [Benson and Curtis 1972; Kilmoyer and Solomon 1973; Lusztig 1979; Alvis and Lusztig 1982], we have $D_{\varphi} \in \mathbb{R}[u]$ for all $\varphi \in \operatorname{Irr}(W)$. The $a$-invariant of $\varphi$ is defined to be the largest $s \geq 0$ such that $u^{s}$ divides the polynomial $D_{\varphi}$. We always have $a_{\varphi} \leq b_{\varphi}$, and $\varphi$ is called special if we have equality.

We define a preorder on $\operatorname{Irr}(W)$ by the condition that $\varphi \leq \varphi^{\prime}$ if and only if $a_{\varphi} \geq a_{\varphi^{\prime}}$. The equivalence relation associated with this preorder will be denoted by $\varphi \sim \varphi^{\prime}$. Thus, we have $\varphi \sim \varphi^{\prime}$ if and only if $a_{\varphi}=a_{\varphi^{\prime}}$. The following result and its proof yield the promised algorithm.

Proposition 2.2. There exist unique elements $p_{\varphi, \varphi^{\prime}} \in$ $\mathbb{Q}(u)$ and $\lambda_{\varphi, \varphi^{\prime}} \in \mathbb{Q}(u)$, where $\varphi, \varphi^{\prime} \in \operatorname{Irr}(W)$, such that the following conditions hold:

1. $\lambda_{\varphi, \varphi^{\prime}}=0$ unless $\varphi \sim \varphi^{\prime}$;

2. $p_{\varphi, \varphi^{\prime}}=0$ unless $\varphi>\varphi^{\prime}$ or $\varphi=\varphi^{\prime}$;

3. $p_{\varphi, \varphi}=u^{a_{\varphi}}$ for all $\varphi$;

4. $\sum_{\varphi_{1}, \varphi_{1}^{\prime} \in \operatorname{Irr}(W)} p_{\varphi, \varphi_{1}} \lambda_{\varphi_{1}, \varphi_{1}^{\prime}} p_{\varphi^{\prime}, \varphi_{1}^{\prime}}=\omega_{\varphi, \varphi^{\prime}}$ for all $\varphi, \varphi^{\prime}$.

The uniqueness is clear. We prove the existence by describing an algorithm for solving the system of equations above. Choose a total ordering on $\operatorname{Irr}(W)$ compatible with the preorder $\geq$ and define matrices of unknowns $P=\left(p_{\varphi, \varphi^{\prime}}\right)$ and $\Lambda=\left(\lambda_{\varphi, \varphi^{\prime}}\right)$. Then the system of equations above says that $P \Lambda P^{\mathrm{tr}}=\Omega$. Moreover, $\Lambda$ is a block diagonal matrix, with blocks corresponding to the equivalences classes under $\sim$, and $P$ is a block lower triangular matrix with diagonal blocks consisting of identity matrices multiplied by $u^{a_{\varphi}}$. Assume we have $r$ blocks, of sizes $n_{1}, \ldots, n_{r}$ and with corresponding $a$-values $a_{1}, \ldots, a_{r}$; partitioning $P, \Lambda, \Omega$ into blocks, the matrix equation 
above has the form

$$
\begin{aligned}
& {\left[\begin{array}{cccc}
I_{1} & 0 & \cdots & 0 \\
P_{2,1} & I_{2} & & \vdots \\
\vdots & & \ddots & 0 \\
P_{r, 1} & \cdots & P_{r, r-1} & I_{r}
\end{array}\right]\left[\begin{array}{cccc}
\Lambda_{1} & 0 & \cdots & 0 \\
0 & \Lambda_{2} & \vdots \\
\vdots & & \ddots & 0 \\
0 & \cdots & 0 & \Lambda_{r}
\end{array}\right]\left[\begin{array}{cccc}
I_{1} & P_{2,1}^{\mathrm{tr}} & \cdots & P_{r, 1}^{\mathrm{tr}} \\
0 & I_{2} & & \vdots \\
\vdots & & \ddots & P_{r, r-1}^{\mathrm{tr}} \\
0 & \cdots & 0 & I_{r}
\end{array}\right] } \\
&=\left[\begin{array}{cccc}
\Omega_{1,1} & \Omega_{1,2} & \cdots & \Omega_{1, r} \\
\Omega_{2,1} & & & \vdots \\
\vdots & & & \Omega_{r-1, r} \\
\Omega_{r, 1} & \cdots & \Omega_{r, r-1} & \Omega_{r, r}
\end{array}\right],
\end{aligned}
$$

where $I_{i}=u^{a_{i}} \mathrm{id}_{n_{i}}$. We can solve this system recursively as follows.

We begin with the first block column. We have $I_{1} \Lambda_{1} I_{1}=\Omega_{1,1}$, which determines $\Lambda_{1}$. For $i>1$ we have $P_{i, 1} \Lambda_{1} I_{1}=\Omega_{i, 1}$. By Lemma 2.1, we know that $\operatorname{det} \Omega_{1,1} \neq 0$. Hence $\Lambda_{1}$ is invertible, and we can determine $P_{i, 1}$. Now consider the $j$-th block column, where $j>1$. Assume that the first $j-1$ block columns of $P$ and the first $j-1$ diagonal blocks of $\Lambda$ have already been determined. We have an equation

$$
I_{j} \Lambda_{j} I_{j}+P_{j, j-1} \Lambda_{j-1} P_{j, j-1}^{\mathrm{tr}}+\cdots+P_{j, 1} \Lambda_{1} P_{j, 1}^{\mathrm{tr}}=\Omega_{j, j},
$$

which can be solved uniquely for $\Lambda_{j}$. In particular, we have now determined all coefficients in $P$ and $\Lambda$ which belong to the first $j$ blocks. We consider the subsystem of equations made up of these blocks; this subsystem looks like the original system written in matrix form above, with $r$ replaced by $j$. By Lemma 2.1, the right hand side has a nonzero determinant. Hence so have the blocks $\Lambda_{1}, \ldots, \Lambda_{j}$. Now we can determine the coefficients of $P$ in the $j$-column: for $i>j$, we have an equation

$P_{i, j} \Lambda_{j} I_{j}+P_{i, j-1} \Lambda_{j-1} P_{j, j-1}^{\mathrm{tr}}+\cdots+P_{i, 1} \Lambda_{1} P_{j, 1}^{\mathrm{tr}}=\Omega_{i, j}$.

Since $\Lambda_{j}$ is invertible, $P_{i, j}$ is determined. Continuing in this way, the system of equations above is solved.

Remark 2.3. Lusztig [1986, § 24] has described a similar algorithm for the computation of (generalized) Green functions of finite reductive groups. But in that case, it is known in advance that solutions exist (since the equations came from orthogonality relations for Green functions). In our case, we had done some experiments in GAP (see Proposition 2.8 and the examples below), and there it always turned out that solutions exist. Lusztig pointed out that to prove this in general, it is necessary to use Lemma 2.1, which he kindly communicated to us.

Remark 2.4. Instead of the preorder $\leq$ defined above, we could have also used any refinement of it such that the equivalence classes are precisely the families of $\operatorname{Irr}(W)$ (in the sense of [Lusztig 1979; 1982]; see also [Lusztig 1984, Chapter 4]). Since the $a$ function is constant on families, this would just yield a finer partition of $\operatorname{Irr}(W)$, but otherwise the algorithm would be the same. But is not clear that the result would also be the same; for this it would be required that the following condition is satisfied:

$\lambda_{\varphi, \varphi^{\prime}}=0$ unless $\varphi, \varphi^{\prime}$ belong to the same family.

In all examples that we computed, this condition turns out to be satisfied.

Similarly to [Lusztig 1986, Theorem 24.8], we expect that the algorithm above actually yields polynomials:

Conjecture 2.5. We have $p_{\varphi, \varphi^{\prime}} \in \mathbb{Z}[u]$ and $\lambda_{\varphi, \varphi^{\prime}} \in \mathbb{Z}[u]$ for all $\varphi, \varphi^{\prime} \in \operatorname{Irr}(W)$. Moreover, the coefficients of $p_{\varphi, \varphi^{\prime}}$ are nonnegative.

To each irreducible character $\varphi$ of $W$, we can associate a rational function $f_{\varphi} \in \mathbb{R}(u)$ by $f_{\varphi}=\lambda_{\varphi, \varphi}$. We expect that the rational functions associated with the special characters will be of particular importance:

Conjecture 2.6. We have $\sum_{\varphi} f_{\varphi}=u^{2 N}$, where the sum is over all special characters $\varphi$ of $W$.

Conjecture 2.7. Assume that $W$ is a Weyl group, and let $G$ be a connected reductive algebraic group such that $W$ is the Weyl group of $G$ with respect to some maximal torus. Assume, moreover, that $F: G \rightarrow$ $G$ is a Frobenius map corresponding to some split $\mathbb{F}_{q}$-rational structure on $G$ (where $q$ is some prime power). Let $\varphi$ be a special character of $W$ and let $\mathcal{C}$ be the corresponding special piece of the unipotent variety of $G$. Then we have $\left|\mathcal{C}^{F}\right|=f_{\varphi}(q)$.

Proposition 2.8. The three conjectures above are true if $(W, S)$ is irreducible of type $A_{n}$ (any $\left.n \geq 1\right), G_{2}$, $F_{4}, E_{6}, E_{7}$, or $E_{8}$. Moreover, condition $(*)$ in Remark 2.4 holds in these cases.

Proof. If $(W, S)$ is of exceptional type, we have used an implementation of our algorithm in GAP and CHEVIE to compute explicitly all elements $p_{\varphi, \varphi^{\prime}}$ 
and $\lambda_{\varphi, \varphi^{\prime}}$. By inspection, Conjectures 2.5 and 2.6, and condition $(*)$ are verified. In order to verify Conjecture 2.7, one has to compare the results of our algorithm with the existing tables of unipotent classes for exceptional Chevalley groups (due to Mizuno and Shoji; see [Carter 1985] for references). In principle, this could be done by hand, but we are indebted to Frank Lübeck for doing this comparison using his data files on a computer.

Finally, let $(W, S)$ be of type $A_{n-1}$ so that the corresponding Chevalley group is $G=\mathrm{GL}_{n}$. In this case, all irreducible characters of $W$ are special, and the special pieces are just the unipotent classes of $G$. Hence it would be sufficient to show that our algorithm produces the same result as that for computing the Green functions of $G$; see [Shoji 1987]. In the latter algorithm, we have to consider a system of matrix equations

$$
Q \Lambda^{\prime} Q^{\operatorname{tr}}=\Omega,
$$

where the matrices $Q, \Lambda^{\prime}$ satisfy similar requirements as in Proposition 2.2 but they are partitioned into blocks of size 1 (since all characters are special). Thus, by the uniqueness of solutions, it is enough to show that $Q, \Lambda^{\prime}$ are automatically partitioned into blocks as required by our algorithm. This is clear for $\Lambda^{\prime}$ (since this is a diagonal matrix). As far as $Q$ is concerned, we must show that if $a_{\varphi}=a_{\varphi^{\prime}}$ (for $\varphi \neq \varphi^{\prime}$ ) then $q_{\varphi, \varphi^{\prime}}=0$. Assume, if possible, that this is not the case. Let $C, C^{\prime}$ be unipotent classes in $G$ such that $\varphi=\varphi_{C}, \varphi^{\prime}=\varphi_{C^{\prime}}$. The condition $\varphi \neq \varphi^{\prime}$ implies $C \neq C^{\prime}$. Now it is known that $q_{\varphi, \varphi^{\prime}}=0$ unless $C$ is contained in the closure of $C^{\prime}$ (see [Shoji 1987, Sect. 5]). But if $a_{\varphi}=a_{\varphi^{\prime}}$, then $\operatorname{dim} C=\operatorname{dim} C^{\prime}$ and hence $C=C^{\prime}$, a contradiction. The positivity of the coefficients of the $p_{\varphi, \varphi^{\prime}}$ asserted in Conjecture 2.5 follows from the corresponding statement for the entries of $Q$; see [Lusztig 1981a, Theorem 1].

We have also checked that the conjectures are true for all Weyl groups of classical types of low rank. For noncrystallographic finite Coxeter groups, the algorithm yields the following results:

Lemma 2.9. Let $W=\langle s, t\rangle$ be a dihedral group such that st has order $m \geq 3$. Then there are precisely three special characters, namely the trivial character $1_{W}$, the sign character $\varepsilon$, and the character $\rho$ of the standard reflection representation. The associated polynomials are given as follows.

\begin{tabular}{lrr}
\hline$\varphi$ & $b_{\varphi}$ & $f_{\varphi}$ \\
\hline$\varepsilon$ & $m$ & 1 \\
$\rho$ & 1 & $\left(u^{m-2}+1\right)\left(u^{m}-1\right)$ \\
$1_{W}$ & 0 & $u^{m-2}\left(u^{2}-1\right)\left(u^{m}-1\right)$ \\
\hline
\end{tabular}

The sum of these three polynomials is $u^{2 m}$, as it should be.

Proof. We solve the system of equations defining $P$ and $\Lambda$ along the lines of the proof of Proposition 2.2. We label the irreducible characters of $W$ such that the first is the sign character, the second the reflection character and the last the trivial character. This ordering is compatible with the preorder introduced above. Then $P, \Lambda$ have the shapes

$P=\left(\begin{array}{ccc}u^{m} & 0 & 0 \\ p & u I_{k-2} & 0 \\ p_{k 1} & q^{\mathrm{tr}} & 1\end{array}\right), \quad \Lambda=\left(\begin{array}{ccc}\lambda_{11} & 0 & 0 \\ 0 & \Lambda_{0} & 0 \\ 0 & 0 & \lambda_{k k}\end{array}\right)$, with $p=\left(p_{21}, \ldots, p_{k-1,1}\right)^{\operatorname{tr}}, q=\left(p_{k 2}, \ldots, p_{k, k-1}\right)^{\operatorname{tr}}$, while

$$
\Omega=\left(\begin{array}{ccc}
u^{2 m} & u^{m} R^{\operatorname{tr}} & u^{m} \\
u^{m} R & \Omega^{\prime} & u^{m} \tilde{R} \\
u^{m} & u^{m} \tilde{R}^{\operatorname{tr}} & u^{2 m}
\end{array}\right)
$$

with

$$
\begin{aligned}
R & =\left(R\left(\varphi_{2}\right), \ldots, R\left(\varphi_{k-1}\right)\right)^{\mathrm{tr}}, \\
\tilde{R} & =\left(R\left(\varphi_{2} \otimes \varepsilon\right), \ldots, R\left(\varphi_{k-1} \otimes \varepsilon\right)\right)^{\mathrm{tr}} .
\end{aligned}
$$

The upper left 2 by 2 block of $P \Lambda P^{\text {tr }}$ equals

$$
\left(\begin{array}{cc}
u^{2 m} \lambda_{11} & u^{m} \lambda_{11} p_{21} \\
u^{m} \lambda_{11} p_{21} & p_{21}^{2} \lambda_{11}+u^{2} \lambda_{22}
\end{array}\right)
$$

while the upper leftmost part of $\Omega$ equals

$$
\left(\begin{array}{cc}
u^{2 m} & u^{m}\left(u^{m-1}+u\right) \\
u^{m}\left(u^{m-1}+u\right) & u^{m}\left(1+u^{m}+u^{m-2}+u^{2}\right)
\end{array}\right)
$$

by the definition of $\omega_{i j}$. The assertion on the first and second line of the table follows.

More generally, this leads to the equations $p=R$, $p_{k 1}=1$ and then

$$
\begin{aligned}
R R^{\operatorname{tr}}+u^{2} \Lambda^{\prime} & =\Omega^{\prime}, \\
R+u \Lambda^{\prime} q & =u^{m} \tilde{R}, \\
1+q^{\operatorname{tr}} \Lambda^{\prime} q+\lambda_{k k} & =u^{2 m} .
\end{aligned}
$$

Clearly this determines $\Lambda^{\prime}=u^{-2}\left(\Omega^{\prime}-R R^{\mathrm{tr}}\right)$. Inserting this into the next equation gives

$$
R+u^{-1}\left(\Omega^{\prime}-R R^{\operatorname{tr}}\right) q=u^{m} \tilde{R} .
$$


We claim that $q=(1,0, \ldots, 0)^{\text {tr }}$ is a solution to this. Then the last equation simplifies to $1+\lambda_{22}+\lambda_{k k}=$ $u^{2 m}$ and the lemma is proved. Thus it remains to check that

$$
\begin{array}{r}
R(\varphi)+u^{m-1} R(\rho \otimes \varphi \otimes \varepsilon)-u^{-1} R(\rho) R(\varphi) \\
=u^{m} R(\varphi \otimes \varepsilon)
\end{array}
$$

for all irreducible characters $\varphi$ of $W$ lying in the same family as $\rho$, i.e., different from 1 and $\varepsilon$. This is an easy exercise.

Example 2.10. Let $(W, S)$ be a Coxeter system of noncrystallographic type $H_{3}$ or $H_{4}$. Using an implementation of the algorithm above in GAP and CHEVIE, we find the following polynomials $f_{\varphi}$ corresponding to special characters $\varphi$. We label an irreducible character of $W$ by a pair $(m, e)$, where $m$ denotes the degree and $e$ is the $b$-invariant. The results are given in Tables 1 and 2; to abbreviate notation, we let $:^{i}$ stand for the factor $u^{i}-1$. The

\begin{tabular}{lr}
\hline$H_{3}$ & $f_{\varphi}$ \\
\hline$\varphi_{1,15}$ & 1 \\
$\varphi_{3,6}$ & $\left(u^{8}+u^{4}+1\right): 10$ \\
$\varphi_{5,5}$ & $u^{4}:^{6}: 10$ \\
$\varphi_{4,3}$ & $u^{8}:^{6}: 10$ \\
$\varphi_{5,2}$ & $u^{10}:^{6}: 10$ \\
$\varphi_{3,1}$ & $u^{10}:^{2}:^{6}: 10$ \\
$\varphi_{1,0}$ & $u^{12}:^{2}:^{6}: 10$ \\
\hline
\end{tabular}

TABLE 1. Polynomials for special pieces in type $H_{3}$. In all the tables and in the text, the notation $:^{i}$

\begin{tabular}{|c|c|}
\hline $\mathrm{H}_{4}$ & $f_{\varphi}$ \\
\hline$\varphi_{1,60}$ & 1 \\
\hline$\varphi_{4,31}$ & $\left(u^{28}+u^{18}+u^{10}+1\right): 30$ \\
\hline$\varphi_{9,22}$ & $u^{10}\left(u^{16}+u^{8}+1\right)::^{20}: 30$ \\
\hline$\varphi_{16,18}$ & $u^{28}\left(u^{6}+1\right)::^{20}:^{30}$ \\
\hline$\varphi_{25,16}$ & $u^{26}:^{12}:^{20}: 30$ \\
\hline$\varphi_{36,15}$ & $u^{38}\left(u^{10}+1\right):^{12}:^{30}$ \\
\hline$\varphi_{24,6}$ & $u^{34}\left(u^{12}+u^{4}+1\right):^{12}:^{20}:^{30}$ \\
\hline$\varphi_{36,5}$ & $u^{48}:^{12}:^{20}:^{30}$ \\
\hline$\varphi_{25,4}$ & $u^{48}:^{2}:^{12}:^{20}:^{30}$ \\
\hline$\varphi_{16,3}$ & $u^{50}:^{2}:^{12}:^{20}:^{30}$ \\
\hline$\varphi_{9,2}$ & $u^{52}:^{2}:^{12}:^{20}$ \\
\hline$\varphi_{4,1}$ & $u^{54}:^{2}:^{12}:^{20}:^{30}$ \\
\hline$\varphi_{1,0}$ & $u^{56}:^{2}:^{12}:^{20}:^{30}$ \\
\hline
\end{tabular}
stands for a factor $u^{i}-1$.

TABLE 2. Polynomials for special pieces in type $H_{4}$. sum of the polynomials $f_{\varphi}$ (for $\varphi$ special) is $u^{30}$ and $u^{120}$, respectively. Moreover, we have checked that condition (*) in Remark 2.4 is satisfied.

We think that the polynomials above for noncrystallographic finite Coxeter groups are those on whose existence was speculated in [Lusztig 1997, (6.10)]. For this note that the first, second and last polynomial in each case coincides with the value predicted in that reference.

Remark 2.11. Lusztig [1997, (6.10)] gives a formula for the size of the special piece corresponding to the special character $\rho \otimes \varepsilon$. Namely, let $d_{1}, \ldots, d_{l}$ be the degrees of $W, m_{1}, \ldots, m_{l}$ the coexponents (see for example [Orlik and Solomon 1980]). Then we should have

$$
f_{\rho \otimes \varepsilon}=\left(u^{h}-1\right) \sum_{i=1}^{l} u^{m_{i}-1}
$$

where $h=\max \left\{d_{1}, \ldots, d_{l}\right\}$ is the Coxeter number of $W$. A short calculation shows that this is the result given by our algorithm if and only if

$$
R(\rho \otimes \rho)=R(\rho)\left(R(\rho)+u^{-1}-u^{h-1}\right) .
$$

This can be checked for the irreducible finite Coxeter groups. Unfortunately we do not see an a priori proof of this formula. (The fake degree of the antisymmetric square $\Lambda^{2}(\rho)$ was computed in [Orlik and Solomon 1980, Corollary 3.2].)

\section{AN EXTENSION}

Recall that our algorithm is a variant of that for computing Green functions. Now the latter admits a generalization to the computation of generalized Green functions; see [Lusztig 1986, §24]. Lusztig suggested that our algorithm should admit a similar generalization.

What we have to do is to consider another Coxeter system $\left(W_{1}, S_{1}\right)$ such that $S$ is a subset of $S_{1}$ and the relations for $W$ are determined from those in $W_{1}$ by the scheme explained in [Lusztig 1993, (1.3)]. The choice of $W_{1}$ is subject to the requirement that the parabolic subgroup of $W_{1}$ generated by $S_{1} \backslash S$ should admit a "cuspidal unipotent character" (see [Lusztig 1993, (2.4)]) and hence a cuspidal family of characters in the sense of [Lusztig 1984, (8.1)]. We 
then consider essentially a similar system of equations as before, but with some modifications taking into account the presence of $W_{1}$.

We define a new matrix $\tilde{\Omega}=\left(\tilde{\omega}_{\varphi, \varphi^{\prime}}\right)_{\varphi, \varphi^{\prime} \in \operatorname{Irr}(W)}$ by

$$
\tilde{\omega}_{\varphi, \varphi^{\prime}}=u^{N_{1}-N} u^{|S|-\left|S_{1}\right|}(u-1)^{\left|S_{1}\right|-|S|} \frac{P_{W_{1}}}{P_{W}} \omega_{\varphi, \varphi^{\prime}},
$$

where $N_{1}$ is the number of reflections in $W_{1}$ and $P_{W_{1}}$ is defined in terms of the degrees of $W_{1}$. (This is analogous to the definition given in [Lusztig 1986, (24.3.4)].)

We also have to modify the $a$-invariants attached to the irreducible characters of $W$. The pair $\left(W, W_{1}\right)$ determines a function $f: S \rightarrow\{1,2, \ldots\}$ such that $f(s)=f(t)$ whenever $s, t \in S$ are conjugate in $W$ (see [Lusztig 1993, 2.4(b)]). We consider the generic Iwahori-Hecke algebra $H^{f}$ defined in a similar way as before, but now the quadratic relations read:

$$
T_{s}^{2}=u^{f(s)} T_{1}+\left(u^{f(s)}-1\right) T_{s} \quad \text { for } s \in S .
$$

Again, we have corresponding generic degrees $D_{\varphi}^{f}$ (which are not necessarily polynomials!). The new $a$-invariants are now defined by

$$
a_{\varphi}^{f}=a_{0}+\left(\text { order of the pole at } u=0 \text { of } D_{\varphi}^{f}\right),
$$

where $a_{0}$ is the (usual) $a$-invariant of the characters belonging to the cuspidal family of characters of the parabolic subgroup of $W_{1}$ generated by $S_{1} \backslash S$.

Taking these data, we can formulate an analogous version of Proposition 2.2, and one might expect that Conjecture 2.5 still holds. We have checked that this is in fact true for all $\left(W, W_{1}\right)$ where $W_{1}$ is a finite Coxeter group of exceptional type. Note that no new cases arise for $W_{1}$ of type $A_{n-1}$.

Example 3.1. Let $\left(W_{1}, S_{1}\right)$ be of type $H_{4}$. According to [Lusztig 1993, §3.3], we have three possibilities such that the requirements for the setting above are satisfied: $(W, S)$ of type $\varnothing, A_{1}$ or $I_{2}(10)$. The first case is trivial; we consider the other two possibilities.

If $(W, S)$ is of type $A_{1}$, the function $f$ takes value 15 , and we have $a_{0}=3$. The modified $a$-invariants of the sign and the trivial character are 18 and 3 , respectively. The matrix $\Lambda$ consists of two $1 \times 1$ blocks with entries $u^{22}::^{12}:{ }^{20}: 30$ and $u^{50}:{ }^{12}:{ }^{20}:{ }^{30}$. We have

$$
P=\left[\begin{array}{cc}
u^{18} & \cdot \\
u^{17} & u^{3}
\end{array}\right]
$$

If $(W, S)$ is of type $I_{2}(10)$, the function $f$ takes values 1,5 , and we have $a_{0}=1$. The modified $a$-invariants are given by

$$
\begin{array}{ccccccccc}
\varphi & \varphi_{1,10} & \varphi_{1,5}^{\prime} & \varphi_{2,1} & \varphi_{2,2} & \varphi_{2,3} & \varphi_{2,4} & \varphi_{1,5}^{\prime \prime} & \varphi_{1,0} \\
a_{\varphi}^{f} & 31 & 22 & 6 & 6 & 6 & 6 & 2 & 1
\end{array}
$$

The matrix $\Lambda$ has 5 blocks, of sizes $1,1,4,1,1$ : the entries are $u^{6}\left(u^{10}+1\right):{ }^{12}:^{30}, u^{14}:{ }^{12}:{ }^{20}:{ }^{30}$,

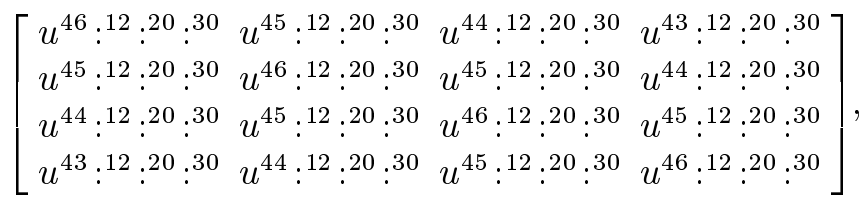

and $u^{52}:^{2}:^{12}:^{30}:^{30}, u^{54}:^{2}:^{12}:^{20}:^{30}$. We have

$$
P=\left[\begin{array}{cccccccc}
u^{31} & \cdot & . & . & . & . & . & . \\
u^{26} & u^{22} & . & . & . & . & . & \cdot \\
u^{30}+u^{22} & u^{18} & u^{6} & . & . & . & . & . \\
u^{29}+u^{23} & u^{19} & . & u^{6} & . & . & . & . \\
u^{28}+u^{24} & u^{20} & . & . & u^{6} & . & . & . \\
u^{27}+u^{25} & u^{21} & . & . & . & u^{6} & . & \cdot \\
u^{26} & . & . & . & . & u^{5} & u^{2} & \cdot \\
u^{21} & u^{17} & u^{5} & . & . & . & . & u
\end{array}\right]
$$

In particular, we see that all entries in these matrices are polynomials.

\section{COMPLEX REFLECTION GROUPS}

Let now $V$ be a complex vector space and $W \subset$ $\mathrm{GL}(V)$ be a finite group generated by pseudoreflections. In order to describe a generalization of the algorithm put forward in the previous section to $W$ we mimic the approach in the real case.

First note that the definition of $R: \mathrm{CF}(W) \rightarrow$ $\mathbb{C}[u]$ given by

$$
R(f):=P_{W}(u-1)^{\operatorname{dim}(V)} \frac{1}{|W|} \sum_{w \in W} \frac{\operatorname{det}_{V}(w) f(w)}{\operatorname{det}_{V}\left(u \cdot \operatorname{id}_{V}-w\right)}
$$

for $f \in \mathrm{CF}(W)$, where $\operatorname{det}_{V}$ denotes the determinant character of $W$ on $V$, makes sense for complex reflection groups and generalizes the definition of $R$ in Section 2. We let $N^{*}$ be the number of pseudoreflections in $W$ and define a matrix $\Omega$ by $\omega_{\varphi, \varphi^{\prime}}:=u^{N^{*}} R\left(\varphi \otimes \varphi^{\prime} \otimes \operatorname{det}_{V}\right)$ as in Section 2.

To define the $a$-invariant of an irreducible character of $W$, we now work with $H=H(W, u)$, the cyclotomic Hecke algebra for $W$ over $\mathbb{C}\left[u, u^{-1}\right]$ with one single parameter $u$ (see [Broué and Malle 1993; Broué et al. 1999]). Let $K$ be a sufficiently large 
extension of $\mathbb{C}(u)$ and $H_{K}$ the algebra obtained by extending scalars from $\mathbb{C}\left[u, u^{-1}\right]$ to $K$. A deformation argument shows again that we have a bijection, $\varphi \leftrightarrow \varphi_{u}$, between the irreducible characters of $W$ and those of $H_{K}$. The definition of generic degrees is more subtle in the present situation: it is conjectured in [Broué and Malle 1993; Broué et al. 1999] (and has now been proved in [Malle and Mathas 1998] for all but finitely many irreducible $W$ ) that $H$ carries a canonical symmetrizing form $\tau: H \rightarrow$ $\mathbb{C}\left[u, u^{-1}\right]$, which in particular vanishes on all elements of a suitable basis (except the identity element), and which specializes to the usual trace form on the group ring of $W$. Hence, in a similar way as before, we see that every irreducible character of $H_{K}$ appears in $\tau$ with nonzero multiplicity, and we may define generic degrees by the equation:

$$
\tau=\sum_{\varphi \in \operatorname{Irr}(W)} \frac{P_{W}}{D_{\varphi}} \varphi_{u}
$$

Assume that $W$ is irreducible and generated by

$$
\operatorname{dim} V=n
$$

reflections of order 2. Then it is expected that $D_{\varphi}$ is a polynomial in $\mathbb{C}[u]$. We can then define $a_{\varphi}$ to be the precise power of $u$ dividing $D_{\varphi}$. A character $\varphi \in \operatorname{Irr}(W)$ is called special if $a_{\varphi}$ is also the precise power of $u$ dividing $R(\varphi)$.

Let $W$ be an irreducible complex reflection group satisfying the assumptions made above. Then either $W$ is real, or $W=G(e, e, n)$ for some $e \geq 3$, $n \geq 3$, (here, the special characters have been identified in [Malle 1995, Lemma 5.16]), or $W$ is one of the primitive complex reflection groups $G_{i}$, for $i \in\{24,27,29,33,34\}$, in the notation of [Shephard and Todd 1954]. For such $W$ the algorithm put forward in Section 2 still makes perfect sense. We believe that the analogues of Conjectures 2.5 and 2.6 remain valid in this more general situation.

Example 4.1. We have used an implementation of the algorithm in GAP and CHEVIE to verify the conjectures on all the primitive complex reflection groups $G_{i}, i \in\{24,27,29,33,34\}$. The $a$-values of the irreducible characters of these groups were determined in [Malle 1999]. Our algorithm yields polynomial entries for $P$ and $\Lambda$. The diagonal entries of $\Lambda$ corresponding to special characters (the lengths of the special pieces) are collected in Tables 3-5. Their sums equal $u^{42}, u^{90}, u^{80}, u^{90}, u^{252}$ respectively. Note that for each of the complex reflection groups above, the size of the second special piece is again given by the formula in Remark 2.11. Here, the special irreducible characters are labeled by pairs $(m, e)$, where $m$ denotes the degree and $e$ is the $a$-invariant.

\section{ACKNOWLEDGEMENTS}

We thank George Lusztig for informing us about Lemma 2.1. We also thank Toshiaki Shoji for several remarks concerning a possible proof of our conjectures for the case of Weyl groups in the framework of character sheaves; see [Shoji 1998].

We started work on this paper while participating in the special semester on representations of alge-

\begin{tabular}{|c|c|c|}
\hline \multirow[b]{2}{*}{$G_{24}$} & & $G_{27}$ \\
\hline & & $\begin{array}{l}\varphi_{1,45} \\
\varphi_{3,16}\end{array} \quad\left(u^{24}+u^{18}+1\right):^{1}$ \\
\hline$\varphi_{1,21}$ & 1 & $\varphi_{10,12} \quad u^{18}\left(u^{6}+1\right):^{12}:^{30}$ \\
\hline$\varphi_{3,8}$ & \multirow{6}{*}{$\begin{array}{r}\left(u^{10}+u^{8}+1\right):{ }^{14} \\
u^{8}\left(u^{2}+1\right):^{6}:^{14} \\
u^{12}\left(u^{2}+1\right):^{6}:{ }^{14} \\
u^{12}:^{4}:^{6}:{ }^{14} \\
u^{14}:^{4}:^{6}:^{14} \\
u^{18}:^{4}:^{6}:^{14}\end{array}$} & $\varphi_{9,9} \quad u^{30}:^{12}: 30$ \\
\hline$\varphi_{7,6}$ & & $\varphi_{15,8} \quad u^{30}:^{12}: 30$ \\
\hline$\varphi_{8,4}$ & & $u^{30}:^{6}:^{12}:^{30}$ \\
\hline$\varphi_{7,3}$ & & $u^{30}:^{6}:^{12}:^{30}$ \\
\hline$\varphi_{3,1}$ & & 0 \\
\hline \multirow[t]{19}{*}{$\varphi_{1,0}$} & & $u^{36}:^{6}:^{12}:^{30}$ \\
\hline & & $\begin{array}{r}0 \\
42.6 .12 .30\end{array}$ \\
\hline & & $\varphi_{1,0} \quad u^{42}:^{6}:^{12}:^{30}$ \\
\hline & \multicolumn{2}{|l|}{$G_{29}$} \\
\hline & $\varphi_{1,40}$ & 1 \\
\hline & $\varphi_{4,21}$ & $\left.+u^{12}+u^{8}+1\right):^{20}$ \\
\hline & $\varphi_{10,18}$ & $u^{8}\left(u^{4}+1\right):^{12}:^{20}$ \\
\hline & $\varphi_{16,13}$ & $2 u^{16}\left(u^{4}+1\right):^{12}:^{20}$ \\
\hline & $\varphi_{15,12}$ & $u^{24}:^{12}:^{20}$ \\
\hline & $\varphi_{15,12}$ & $u^{16}:^{8}:^{12}:^{20}$ \\
\hline & $\varphi_{20,9}$ & $2 u^{20}:^{8}:^{12}:^{20}$ \\
\hline & $\varphi_{24,6}$ & $u^{24}\left(u^{4}+1\right):^{8}:^{12}:^{20}$ \\
\hline & $\varphi_{20,5}$ & $u^{28}:^{8}:^{12}:^{20}$ \\
\hline & $\varphi_{15,4}$ & $u^{28}:^{4}:^{8}:^{12}:^{20}$ \\
\hline & $\varphi_{15,4}$ & $u^{32}:^{8}:^{12}:^{20}$ \\
\hline & $\varphi_{16,3}$ & 0 \\
\hline & $\varphi_{10,2}$ & $u^{32}:^{4}:^{8}:^{12}:^{20}$ \\
\hline & $\varphi_{4,1}$ & 0 \\
\hline & $\varphi_{1,0}$ & $u^{36}:^{4}:^{8}:^{12}:^{20}$ \\
\hline
\end{tabular}
braic groups and related finite groups at the Isaac

TABLE 3. Polynomials for special pieces in type $G_{24}$, $G_{27}$, and $G_{29}$. 


\begin{tabular}{|c|c|}
\hline$G_{33}$ & \\
\hline$\varphi_{1,45}$ & 1 \\
\hline$\varphi_{5,28}$ & $\left(u^{14}+u^{12}+u^{8}+u^{6}+1\right):^{18}$ \\
\hline$\varphi_{15,23}$ & $u^{6}\left(u^{10}+u^{8}+u^{6}+u^{4}+u^{2}+1\right):^{10}:^{18}$ \\
\hline$\varphi_{30,18}$ & $u^{10}\left(u^{16}+u^{14}+2 u^{12}+2 u^{10}+u^{8}+u^{6}+u^{4}-1\right):^{10}:^{18}$ \\
\hline$\varphi_{30,13}$ & $u^{14}\left(u^{8}+2 u^{6}+u^{4}+u^{2}+1\right):^{10}:^{12}:^{18}$ \\
\hline$\varphi_{15,12}$ & $u^{26}:^{10}:^{12}:^{18}$ \\
\hline$\varphi_{81,11}$ & $u^{22}\left(u^{6}+2 u^{4}+2 u^{2}+1\right):^{10}:^{12}:^{18}$ \\
\hline$\varphi_{60,10}$ & $u^{22}\left(u^{2}+1\right):^{6}: 10:^{12}:^{18}$ \\
\hline$\varphi_{45,10}$ & $u^{24}:^{4}:^{10}:^{12}:^{18}$ \\
\hline$\varphi_{15,9}$ & $u^{28}:^{4}:^{10}:^{12}:^{18}$ \\
\hline$\varphi_{64,8}$ & $u^{26}\left(u^{2}+1\right):^{6}:^{10}:^{12}:^{18}$ \\
\hline$\varphi_{60,7}$ & $u^{28}\left(u^{2}+1\right):^{6}:^{10}:^{12}:^{18}$ \\
\hline$\varphi_{45,7}$ & $u^{26}\left(u^{2}+1\right):^{6}:^{10}:^{12}:^{18}$ \\
\hline$\varphi_{81,6}$ & $u^{28}:^{4}:^{6}:^{10}:^{12}:^{18}$ \\
\hline$\varphi_{30,4}$ & $u^{28}\left(u^{2}+1\right):^{4}:^{6}:^{10}:^{12}:^{18}$ \\
\hline$\varphi_{30,3}$ & $u^{36}\left(u^{2}+1\right):^{6}:^{10}:^{12}:^{18}$ \\
\hline$\varphi_{15,2}$ & $u^{36}:^{4}:^{6}:^{10}:^{12}:{ }^{18}$ \\
\hline$\varphi_{5,1}$ & 0 \\
\hline$\varphi_{1,0}$ & $u^{40}:^{4}:^{6}:^{10}:^{12}:^{18}$ \\
\hline
\end{tabular}

TABLE 4. Polynomials for special pieces in type $G_{33}$.

Newton Institute (Cambridge, U.K.) from January to July 1997. It is a pleasure to thank the organisers, Michel Broué, Roger Carter and Jan Saxl, for this invitation and the Isaac Newton Institute for its hospitality.

The paper was completed while the first author was holding a CNRS research position at the University Paris VII (France).

\section{ELECTRONIC AVAILABILITY}

A file containing the programs and examples mentioned at the end of Section 1 can be obtained from the 'contributions' directory of the home page of CHEVIE under http://www.math.rwth-aachen.de/ $\sim$ CHEVIE.

\section{REFERENCES}

[Alvis and Lusztig 1982] D. Alvis and G. Lusztig, "The representations and generic degrees of the Hecke algebra of type $\mathrm{H}_{4}$ ", J. Reine Angew. Math. 336 (1982), 201-212. Correction in 449 (1994), 217-218.

[Benson and Curtis 1972] C. T. Benson and C. W. Curtis, "On the degrees and rationality of certain characters of finite Chevalley groups", Trans. Amer. Math. Soc. 165 (1972), 251-273. Corrections and additions by C. W. Curtis in 202 (1975), 405-406.

\begin{tabular}{|c|c|}
\hline$G_{34}$ & \\
\hline$\varphi_{1,126}$ & 1 \\
\hline$\varphi_{6,85}$ & $\left(u^{36}+u^{30}+u^{24}+u^{18}+u^{12}+1\right):^{42}$ \\
\hline$\varphi_{21,68}$ & $u^{12}\left(u^{30}+2 u^{24}+2 u^{18}+2 u^{12}+u^{6}+1\right):^{30}:^{42}$ \\
\hline$\varphi_{56,57}$ & $u^{24}\left(u^{42}+2 u^{36}+3 u^{30}+3 u^{24}+u^{18}-u^{6}-1\right):^{30}:^{42}$ \\
\hline$\varphi_{105,46}$ & $u^{36}\left(2 u^{24}+4 u^{18}+4 u^{12}+3 u^{6}+1\right):^{24}:^{30}:^{42}$ \\
\hline$\varphi_{70,45}$ & $u^{66}:^{24}:^{30}: 42$ \\
\hline$\varphi_{126,41}$ & $u^{42}\left(u^{12}+2 u^{6}+1\right):^{18}:^{24}:^{30}:^{42}$ \\
\hline$\varphi_{315,36}$ & $u^{54}\left(u^{30}+3 u^{24}+4 u^{18}+2 u^{12}-1\right):^{24}:^{30}:^{42}$ \\
\hline$\varphi_{420,31}$ & $u^{60}\left(3 u^{12}+3 u^{6}+1\right):^{18}:^{24}:^{30}:^{42}$ \\
\hline$\varphi_{210,30}$ & $u^{78}:^{18}:^{24}:^{30}:^{42}$ \\
\hline$\varphi_{384,29}$ & $u^{72}\left(u^{6}+1\right):^{18}:^{24}:^{30}:^{42}$ \\
\hline$\varphi_{315,28}$ & $u^{66}\left(u^{12}+u^{6}+1\right):^{18}:^{24}:^{30}:^{42}$ \\
\hline$\varphi_{560,27}$ & $u^{66}\left(u^{6}+1\right):^{12}:^{18}:^{24}:^{30}:^{42}$ \\
\hline$\varphi 729,24$ & $u^{78}\left(u^{12}+3 u^{6}+2\right):^{18}:^{24}:^{30}:^{42}$ \\
\hline$\varphi_{840,23}$ & $u^{72}\left(u^{6}+1\right):^{12}:^{18}:^{24}:^{30}:^{42}$ \\
\hline$\varphi_{630,23}$ & $u^{78}:^{12}:^{18}:^{24}:^{30}:^{42}$ \\
\hline$\varphi_{896,21}$ & $u^{84}:^{12}:^{18}:^{24}:^{30}:^{42}$ \\
\hline$\varphi_{630,20}$ & $u^{84}:^{12}:^{18}:^{24}:^{30}: 42$ \\
\hline$\varphi_{840,19}$ & $u^{78}\left(2 u^{6}+1\right):^{12}:^{18}:^{24}:^{30}:^{42}$ \\
\hline$\varphi_{560,18}$ & $u^{96}\left(u^{6}+1\right):^{18}:^{24}:^{30}:^{42}$ \\
\hline$\varphi_{1280,15}$ & $u^{84}\left(u^{12}+2 u^{6}-1\right):^{12}:^{18}:^{24}:^{30}:^{42}$ \\
\hline$\varphi_{630,14}$ & $u^{96}:^{12}:^{18}:^{24}:^{30}:^{42}$ \\
\hline$\varphi_{840,13}$ & $2 u^{96}:^{12}:^{18}:^{24}:^{30}:^{42}$ \\
\hline$\varphi_{896,12}$ & $u^{96}:^{6}:^{12}:^{18}:^{24}:^{30}:^{42}$ \\
\hline$\varphi_{210,12}$ & $u^{102}:^{12}:^{18}:^{24}:^{30}:^{42}$ \\
\hline$\varphi 840,11$ & $u^{102}:^{12}:^{18}:^{24}:^{30}:^{42}$ \\
\hline$\varphi 630,11$ & $u^{96}:^{6}:^{12}:^{18}:^{24}:^{30}:^{42}$ \\
\hline$\varphi_{729,10}$ & 0 \\
\hline$\varphi_{315,10}$ & 0 \\
\hline$\varphi_{560,9}$ & $u^{102}:^{6}:^{12}:^{18}:^{24}:^{30}:^{42}$ \\
\hline$\varphi_{70,9}$ & $u^{108}:^{12}:^{18}:^{24}:^{30}:^{42}$ \\
\hline$\varphi_{384,8}$ & 0 \\
\hline$\varphi_{420,7}$ & $u^{102}:^{6}:^{12}:^{18}:^{24}:^{30}:^{42}$ \\
\hline$\varphi_{315,6}$ & $u^{108}:^{6}:^{12}:^{18}:^{24}:^{30}:^{42}$ \\
\hline$\varphi_{126,5}$ & 0 \\
\hline$\varphi_{105,4}$ & $u^{108}:^{6}:^{12}:^{18}:^{24}:^{30}:^{42}$ \\
\hline$\varphi_{56,3}$ & $u^{114}:^{6}:^{12}:^{18}:^{24}:^{30}:^{42}$ \\
\hline$\varphi_{21,2}$ & 0 \\
\hline$\varphi_{6,1}$ & 0 \\
\hline$\varphi_{1,0}$ & $u^{120}:^{6}:^{12}:^{18}:^{24}:^{30}:^{42}$ \\
\hline
\end{tabular}

TABLE 5. Polynomials for special pieces in type $G_{34}$.

[Bourbaki 1968] N. Bourbaki, Groupes et algèbres de Lie, Chap. IV, V, VI, Actualités Scientifiques et Industrielles 1337, Hermann, Paris, 1968.

[Broué and Malle 1993] M. Broué and G. Malle, "Zyklotomische Heckealgebren", pp. 119-189 in Représentations unipotentes génériques et blocs des groupes réductifs finis, edited by M. Broué et al., Astérisque 212, Soc. math. France, Paris, 1993. 
[Broué et al. 1999] M. Broué, G. Malle, and J. Michel, "Towards Spetses", preprint, 1999. To appear in Transformation Groups.

[Carter 1985] R. W. Carter, Finite groups of Lie type: Conjugacy classes and complex characters, John Wiley, New York, 1985.

[Curtis and Reiner 1987] C. W. Curtis and I. Reiner, Methods of representation theory, II, John Wiley, New York, 1987.

[Geck and Malle 1999] M. Geck and G. Malle, "On the existence of a unipotent support for the irreducible characters of a finite group of Lie type", Trans. Amer. Math. Soc. (1999).

[Geck et al. 1996] M. Geck, G. Hiss, F. Lübeck, G. Malle, and G. Pfeiffer, "CHEVIE - a system for computing and processing generic character tables", Appl. Algebra Engrg. Comm. Comput. 7:3 (1996), 175210.

[Kilmoyer and Solomon 1973] R. Kilmoyer and L. Solomon, "On the theorem of Feit-Higman", J. Combinatorial Theory Ser. A 15 (1973), 310-322.

[Lusztig 1979] G. Lusztig, "A class of irreducible representations of a Weyl group", Nederl. Akad. Wetensch. Indag. Math. 41:3 (1979), 323-335.

[Lusztig 1981a] G. Lusztig, "Green polynomials and singularities of unipotent classes", Adv. in Math. 42:2 (1981), 169-178.

[Lusztig 1981b] G. Lusztig, "On a theorem of Benson and Curtis", J. Algebra 71:2 (1981), 490-498.

[Lusztig 1982] G. Lusztig, "A class of irreducible representations of a Weyl group, II", Nederl. Akad. Wetensch. Indag. Math. 44:2 (1982), 219-226.

[Lusztig 1984] G. Lusztig, Characters of reductive groups over a finite field, Annals of Math. Studies 107, Princeton University Press, Princeton, N.J., 1984.

[Lusztig 1986] G. Lusztig, "Character sheaves, V", Adv. in Math. 61:2 (1986), 103-155. Erratum in 62:3 (1986), 313-314.
[Lusztig 1993] G. Lusztig, "Appendix: Coxeter groups and unipotent representations", pp. 191-203 in Représentations unipotentes génériques et blocs des groupes réductifs finis, edited by M. Broué et al., Astérisque 212, Soc. math. France, Paris, 1993.

[Lusztig 1997] G. Lusztig, "Notes on unipotent classes", Asian J. Math. 1:1 (1997), 194-207.

[Malle 1995] G. Malle, "Unipotente Grade imprimitiver komplexer Spiegelungsgruppen", J. Algebra 177:3 (1995), 768-826.

[Malle 1999] G. Malle, "On the generic degrees of cyclotomic algebras", preprint, 1999.

[Malle and Mathas 1998] G. Malle and A. Mathas, "Symmetric cyclotomic Hecke algebras", J. Algebra 205:1 (1998), 275-293.

[Orlik and Solomon 1980] P. Orlik and L. Solomon, "Unitary reflection groups and cohomology", Invent. Math. 59:1 (1980), 77-94.

[Schönert et al. 1994] M. Schönert et al., GAP: Groups, algorithms, and programming, 4th ed., Lehrstuhl D für Mathematik, RWTH Aachen, 1994. See ftp:// dimacs.rutgers.edu/pub/ or http://www-gap.dcs. st-and.ac.uk/ gap.

[Shephard and Todd 1954] G. C. Shephard and J. A. Todd, "Finite unitary reflection groups", Canadian J. Math. 6 (1954), 274-304.

[Shoji 1987] T. Shoji, "Green functions of reductive groups over a finite field", pp. 289-301 in The Arcata Conference on Representations of Finite Groups (Arcata, CA, 1986), Amer. Math. Soc., Providence, RI, 1987.

[Shoji 1998] T. Shoji, "Green functions and a conjecture of Geck and Malle", preprint, 1998. To appear in Beiträge zur Algebra und Geometrie.

[Spaltenstein 1982] N. Spaltenstein, Classes unipotentes et sous-groupes de Borel, LNM 946, Springer, Berlin, 1982.

Meinolf Geck, Institut Girard Desargues, bât. 101, Université Lyon 1, 43 boulevard du 11 novembre 1918, 69622 Villeurbanne cedex, France (geck@desargues.univ-lyon1.fr)

Gunter Malle, Fachbereich Mathematik/Informatik, Heinrich-Plett-Straße 40, D 34132 Kassel, (malle@mathematik.uni-kassel.de)

Received December 2, 1997; accepted in revised form August 27, 1998 